Mens

Revue d'histoire intellectuelle et culturelle

mens

\title{
La Grande Noirceur encore et toujours
}

\section{Gilles Bourque}

Volume 18, numéro 2, printemps 2018

La Grande Noirceur

URI : https://id.erudit.org/iderudit/1066260ar

DOI : https://doi.org/10.7202/1066260ar

Aller au sommaire du numéro

Éditeur(s)

Centre de recherche en civilisation canadienne-française

ISSN

1492-8647 (imprimé)

1927-9299 (numérique)

Découvrir la revue

Citer cet article

Bourque, G. (2018). La Grande Noirceur encore et toujours. Mens, 18(2), 39-65. https://doi.org/10.7202/1066260ar

\section{Résumé de l'article}

Près de soixante années après l'avènement de la Révolution tranquille, la notion de Grande Noirceur continue de hanter la mémoire du duplessisme. Ce texte examine d'abord les différentes approches que des essayistes, des historiens et des sociologues ont implicitement ou explicitement mises en oeuvre dans leurs travaux ou leurs réflexions sur la notion de Grande Noirceur. On peut, à ce propos, distinguer trois postures fort différentes. Les deux premières, la réitération et la rectification, prennent en quelque sorte la notion au pied de la lettre et cherchent soit à en réaffirmer la validité, soit, tout au contraire, à la contester en montrant sa fausseté. La troisième approche, celle de l'objectivation, consiste à construire la notion de Grande Noirceur en s'inspirant des règles de la méthode dans le domaine des sciences sociales. Dans une telle perspective, l'auteur proposera de déplacer le regard vers les années 1960 et 1970 et de considérer l'idée de Grande Noirceur comme l'une des notions centrales du discours providentialiste québécois. Ce discours s’organise, en effet, à partir de deux notions antithétiques, celles de Grande Noirceur et de Révolution tranquille, dont il est impossible de faire la synthèse. À partir de là, le discours providentialiste se déploie en deux chaînes de significations parfaitement étrangères l'une à l'autre. L'article se termine par un bref retour sur le duplessisme.
Ce document est protégé par la loi sur le droit d'auteur. L'utilisation des services d’Érudit (y compris la reproduction) est assujettie à sa politique d'utilisation que vous pouvez consulter en ligne.

https://apropos.erudit.org/fr/usagers/politique-dutilisation/ 


\title{
La Grande Noirceur encore et toujours
}

\author{
Gilles Bourque \\ Professeur émérite \\ Département de sociologie \\ Université du Québec à Montréal
}

\section{Résumé}

Près de soixante années après l'avènement de la Révolution tranquille, la notion de Grande Noirceur continue de hanter la mémoire du duplessisme. Ce texte examine d'abord les différentes approches que des essayistes, des historiens et des sociologues ont implicitement ou explicitement mises en œuvre dans leurs travaux ou leurs réflexions sur la notion de Grande Noirceur. On peut, à ce propos, distinguer trois postures fort différentes. Les deux premières, la réitération et la rectification, prennent en quelque sorte la notion au pied de la lettre et cherchent soit à en réaffirmer la validité, soit, tout au contraire, à la contester en montrant sa fausseté. La troisième approche, celle de l'objectivation, consiste à construire la notion de Grande Noirceur en s'inspirant des règles de la méthode dans le domaine des sciences sociales. Dans une telle perspective, l'auteur proposera de déplacer le regard vers les années 1960 et 1970 et de considérer l'idée de Grande Noirceur comme l'une des notions centrales du discours providentialiste québécois. Ce discours s'organise, en effet, à partir de deux notions antithétiques, celles de Grande Noirceur et de Révolution tranquille, dont il est impossible de faire la synthèse. À partir de là, le discours providentialiste se déploie en deux chaînes 
de significations parfaitement étrangères l'une à l'autre. L'article se termine par un bref retour sur le duplessisme.

Dans les travaux que j'ai réalisés avec d'autres collègues sur le duplessisme, il n’a jamais été question de nous référer à la notion de Grande Noirceur, soit pour la conforter, soit pour l'invalider ${ }^{1}$. Il nous semblait élémentaire, d'un strict point de vue méthodologique, de rejeter l'idée de soumettre l'analyse de la période à la représentation qu’a imposée le discours triomphant de la Révolution tranquille. Lors de la publication de nos ouvrages, principalement à la suite de la parution de La société libérale duplessiste, nous n'allions cependant pas échapper aussi facilement au grand récit des années $1960^{2}$. Nous avons été soupçonnés par plusieurs d'œuvrer sans le dire à la réhabilitation du régime tant honni. Nous avons soutenu et croyons avoir montré que le pouvoir duplessiste assurait son hégémonie grâce à la formulation et à la diffusion d'un discours politique autoritaire et disciplinaire d'inspiration libéralo-traditionaliste. Nous avons illustré cette thèse en montrant la centralité de la notion de progrès et l'importance de celle de tradition. "Restons traditionnels et progressifs ", proclamait le ministre des Finances de l'Union nationale 3 .

Un vif débat a suivi la publication de cet ouvrage sur lequel il est hors de question de revenir ici. Retenons seulement qu'un colloque a été organisé à la suite duquel un livre a paru, intitulé Duplessis : entre la Grande Noirceur et la société libérale. La Grande Noirceur, encore et toujours! Nous croyions l'avoir congédiée, mais voilà qu'elle revenait par la porte arrière!

${ }^{1}$ Gilles Bourque, Jules Duchastel et Jacques Beauchemin, La société libérale duplessiste, Montréal, Les Presses de l'Université de Montréal, 1994; Gilles Bourque et Jules Duchastel, Restons traditionnels et progressifs, Montréal, Éditions du Boréal, 1988; Gilles Bourque et Anne Legaré, Le Québec, la question nationale, Paris, François Maspero, 1979, p. 141-165.

2 Bourque, Duchastel et Beauchemin, La société libérale duplessiste.

3 Bourque et Duchastel, Restons traditionnels et progressifs.

4 Alain-G. Gagnon et Michel Sarra-Bournet (dir.), Duplessis : entre la Grande Noirceur et la société libérale, Montréal, Québec Amérique, 1997. 
Bien sûr, on nous a accusés de révisionnisme, nous en devenions même "le meilleur exemple ". Heureusement, nous n'étions pas seuls, le même auteur a suggéré que, dans Québec 1945-2000 : les intellectuels et le temps de Duplessis, tome $2^{6}$, Léon Dion était "influencé fortement par le contexte néoconservateur en vogue depuis la crise économique de $1981^{7} »$. Tout se passait comme s'il fallait choisir son camp et ne jamais transgresser la frontière entre l'avant et l'après, le passé et le présent, les ténèbres et la lumière. Cette opposition binaire s'est imposée comme une doxa au début des années 1960, même les cancres l'ont compris. Ainsi, durant les années 1990, en réponse à une question sur la Révolution tranquille posée lors d'un examen de mi-session, un étudiant a commencé par écrire : «Avant 1960, c'était le néant ». Au cours suivant, commentant l'examen, j'ai signalé à mes étudiants qu'un tel énoncé était rigoureusement faux et leur en ai donné pour preuve le fait indubitable que, durant les années 1940 et 1950 , j'étais déjà vivant! Toute tragi-comique qu'elle est, cette anecdote n'en permet pas moins de souligner que la notion de Grande Noirceur pose la question de la relation entre ce qu'on appelait naguère l'idéologie et les rapports réels et que l'on pourrait aussi saisir comme les liens entre le discours et les pratiques non discursives ou entre la fiction et la vérité.

Dans cet article, je commencerai par examiner les différentes approches que des essayistes, des historiens et des sociologues ont implicitement ou explicitement mises en ouvre dans leurs travaux ou leurs réflexions incidentes sur la Grande Noirceur. Dans une deuxième partie, j'avancerai la thèse que la Grande Noirceur est une notion, un mythe ou un mythistoire, comme on voudra, qui fait partie intégrante du discours politique des années 1960 et, qu’à ce

5 Michael D. Behiels, «Duplessis, le duplessisme et la prétendue reconstitution du passé ", dans Gagnon et Sarra-Bournet (dir.), Duplessis : entre la Grande Noirceur et la société libérale, p. 320.

${ }^{6}$ Léon Dion, Québec 1945-2000 : les intellectuels et le temps de Duplessis, t. 2, Québec, Les Presses de l'Université Laval, 1993.

7 Behiels, «Duplessis », p. 320. 
titre, son intérêt demeure plus que relatif dans l'étude des années 1940 et 1950. Je plaiderai enfin en faveur du développement d'une problématique qui, dans l'analyse du duplessisme, fait l'économie de l’imaginaire dominant la période qui lui a succédé.

\section{Que faire de la Grande Noirceur?}

De quoi parle donc le locuteur lorsqu'il emploie la notion de Grande Noirceur ou, pourrait-on aussi nous demander, qu'entendent les milliers et les milliers de récepteurs lorsque, depuis les années 1960, ils s'y réferent? Au niveau le plus élémentaire, on pourrait dire qu'il s'agit d'une locution dont le référent renvoie de façon entièrement négative à cette période de l'histoire du Québec qui commence en 1944 et se termine en 1960. Que faire cependant de cette évidence? On peut la recevoir comme une vérité à laquelle on adhérera spontanément ou, au contraire, comme une fausseté qu'on s'empressera de dénoncer. Une troisième possibilité s'offre heureusement à nous. Il s'agira de mettre en pratique les règles de la méthode en sciences sociales en commençant par construire la notion de Grande Noirceur comme un objet d'analyse. Penchons-nous sur ces trois approches fort différentes.

\section{La réitération}

Dès le début des années 1960, la cause était entendue et le verdict rendu. Le Québec avait eu jusque-là des allures moyenâgeuses, et le duplessisme représentait un régime obscurantiste, autoritaire et réactionnaire, inspiré d'une idéologie clérico-nationaliste scellant l'alliance entre l'Église et l'État. La Révolution tranquille, heureusement, avait enfin permis au Québec d'accéder à la modernité et de sortir de la Grande Noirceur. À partir des années 1980, des historiens, des sociologues et des politologues ont voulu dépasser ces fausses évidences et renouveler l'analyse de l'histoire du Québec précédant la Révolution tranquille. Dès lors, la modernité commença 
à reculer dans l'histoire ${ }^{8}$. Comme je l'ai souligné d'entrée de jeu, c'est cependant la volonté d'étudier le duplessisme en contournant en quelque sorte la notion ou le mythe de la Grande Noirceur et sans doute encore davantage l'idée d'utiliser d'une quelconque façon le qualificatif « libéral» qui créèrent le plus de remous 9 .

Après une période d'incrédulité, plusieurs décidèrent de réagir en réitérant les poncifs de la doxa des années 1960. Des acteurs de l'époque décidèrent d'intervenir comme, par exemple, Madeleine Parent, Gérard Pelletier et Jacques Hébert ${ }^{10}$. "Oui, oui, vous avez raison : j'en ai gros sur la patate! Comme tous ceux qui ont vécu la Grande Noirceur ${ }^{11}$ ".

Les auteurs reprennent essentiellement les analyses qu'ils avaient formulées durant les années 1950. Or, il faut le souligner, ces textes constituent précisément la matière première à l'origine de la formulation de la notion de Grande Noirceur. La réitération ne saurait être plus claire que dans le livre de Jacques Hébert. La période 19441960 était, selon lui, " cette tranche particulièrement noire de la Grande Noirceur qui n'en finissait plus de finir ${ }^{12}$ ». Aussi bien le régime que l'homme ne sauraient trouver grâce à ses yeux : "Là encore les convictions profondes de Duplessis, c'est-à-dire ses idées préconçues les plus réactionnaires, moyenâgeuses, obscurantistes

8 Paul-André Linteau, et al., Histoire du Québec contemporain : le Québec depuis 1930, t. 2, Montréal, Éditions du Boréal, 1989; Yvan Lamonde et Esther Trépanier, L'avènement de la modernité au Québec, Québec, Institut québécois de recherche sur la culture, 1986.

9 Alors que Léon Dion propose le concept de libéralisme conservateur (voir Dion, Québec 1945-1960), nous avons proposé ceux de libéralo-traditionalisme et de société libérale (voir Bourque, Duchastel et Beauchemin, La société libérale duplessiste; Bourque et Duchastel, Restons traditionnels et progressifs).

10 Voir Madeleine Parent, "Témoignage ", dans Gagnon et Sarra-Bournet (dir.), Duplessis : entre la Grande Noirceur et la société libérale, p. 17-20; Gérard Pelletier, "Témoignage ", dans Gagnon et Sarra-Bournet (dir.), Duplessis : entre la Grande Noirceur et la société libérale, p. 21-25; Jacques Hébert, Duplessis, non merci, Montréal, Éditions du Boréal, 2000.

11 Ibid., p. 182.

12 Ibid., p. 61. 
correspondent assez bien à celles du clergé catholique du Québec ${ }^{13}$ "; "Cet homme seigneur de la Grande Noirceur a personnifié le duplessisme dans toute sa bêtise triomphante ${ }^{14}{ }^{\prime}$. Bref, pour les tenants de la réitération le jugement demeurera toujours sans appel.

\section{La rectification}

La deuxième approche prend le contrepied de la première. Ici la vérité devient fausseté, mais l'on ne s'intéresse guère davantage aux conditions de production du discours, sinon pour avancer une sorte de théorie du complot qui ne mène pas bien loin et n'ouvre guère de perspectives. En voici deux exemples.

Pour Martin Lemay ${ }^{15}$, la Grande Noirceur est un mythe au sens où le définit le Grand Robert de la langue française : "Représentation de faits ou de personnages dont l'existence historique est réelle ou admise, mais qui ont été déformés ou amplifiés par l'imagination collective ${ }^{16} \ldots »$. Lemay retient ainsi une définition de sens commun, caractéristique de la modernité durant laquelle la notion de mythe renvoie nécessairement à une déformation des faits ou de la réalité dans une relation essentiellement univoque. Pour l'auteur, dans le mythe de la Grande Noirceur, la réalité de la gouverne duplessiste a été " déformée et amplifiée négativement ${ }^{17}$ ». En ce sens, " dès que le nom de Duplessis est évoqué [...] se met en branle un dispositif sophistiqué - exécuté prestement par les adeptes du mythe de la Grande Noirceur - dont le but ultime est de le discréditer, quand ce n'est pas de discréditer la société canadienne-française dans son ensemble ${ }^{18}$ ". Double cible du mythe qui, en même temps, cherche "à jeter l'anathème sur le conservatisme " dont les thèmes sont «le

13 Ibid., p. 153.

${ }^{14}$ Ibid., p. 186.

15 Martin Lemay, À la défense de Maurice Duplessis, Montréal, Québec Amérique, 2016.

16 Ibid., p. 33.

17 Ibid., p. 34.

18 Ibid., p. 57. 
respect des traditions, la hiérarchie sociale, le moralisme et la cohésion " et, enfin, "le libéralisme économique ${ }^{19}$ ». Le mythe, selon l'auteur, a pu facilement s'imposer en 1960 parce que les opposants au régime duplessiste " ont envahi tous les lieux de pouvoir et d'influence" alors que les forces qui défendaient « les idées du clergé, les droitistes et les conservateurs se sont littéralement effondrées ${ }^{20}$ ».

Jusqu'ici Lemay décrit de façon relativement classique un rapport de force d'ordre idéologique à la faveur duquel se sont affrontés discours et contre-discours et dont, bien sûr, nous connaissons les vainqueurs. Par la suite, cependant, l'auteur change soudainement de registre et devient par procuration une sorte de protagoniste. Dès lors, il prend parti et n'a de cesse de dénoncer les artisans à l'origine du mythe de la Grande Noirceur. Il attire l'attention en ce sens sur " un trio infernal, une véritable ligue factieuse : Gérard Pelletier, Pierre Elliott Trudeau et Jacques Hébert ${ }^{21}$ ». Il finit même par conclure que les années 1950 ont été l'occasion d'une vaste conspiration! "Cette conspiration a été tellement bien orchestrée que ladite formule (celle de Grande Noirceur) s'est muée en mythe historico-politique immuable : la boucle mystificatrice était solidement "bouclée " pour des siècles et des siècles ${ }^{22}$ ". Heureusement, l'auteur n'attendra pas si longtemps. Il travaillera à la réhabilitation de Duplessis car, pour lui, il s'agit de la seule façon de contrer le mythe de la Grande Noirceur.

Charles-Philippe Courtois emprunte une voie similaire. La Grande Noirceur devient une "légende noire [...] exagérément négative ", construite parce que Duplessis était à la tête d'un gouvernement conservateur ${ }^{23}$. L'auteur étudie les revues Cité libre et Parti pris. La première aurait « joué un rôle central dans la conception

19 Ibid., p. 84.

20 Ibid., p. 23.

${ }^{21}$ Ibid., p. 163.

22 Ibid., p. 27.

${ }^{23}$ Charles-Philippe Courtois, "Cité libre, Duplessis et une vision tronquée du Québec ", dans Xavier Gélinas et Lucia Ferretti (dir.), Duplessis, son milieu, son époque, Québec, Éditions du Septentrion, 2010, p. 54. 
et la diffusion de cette représentation trompeuse ${ }^{24}$ ", alors que les artisans de la seconde « conservent la vision du passé québécois et de la Grande Noirceur forgée par les Cité libristes ${ }^{25}$ ». On pourrait, bien sûr, avancer ici que Parti pris s'inspire bien davantage de la vision de l'histoire du Québec proposée par Maurice Séguin et l'École historique de Montréal, mais tel n'est pas notre propos ${ }^{26}$. Retenons que le projet de Courtois est d'abord et avant tout de montrer « la fausseté de cette description de l'histoire du Québec ${ }^{27}$ ".

Le projet de Lemay et de Courtois ne saurait donc être plus clair : il s'agit pour eux de rectifier la vision de l'histoire avancée dans le « mythe » ou la « légende » de la Grande Noirceur. Les intentions de Gilles Paquet dans ses textes sur la Révolution tranquille ratissent beaucoup plus large. L'auteur se livre à une critique radicale, sinon à une démolition en règle de la configuration des rapports sociaux que s'est donnée le Québec sous l'égide de l'État-providence. Pour des raisons évidentes, je ne retiendrai que sa vision du passé. Tout en affirmant que la période précédant la Révolution tranquille n'est pas une époque de Grande Noirceur ${ }^{28}$, il n'hésite pas à recourir à la théorie du complot :

L'intelligentsia va conspirer pour répandre de fausses représentations : c'est comme si la banque centrale s'était mise à émettre de la fausse monnaie. On va systématiquement, délibérément et abusivement noircir un avant 1960 personnaliste et communautaire, pour délibérément et abusivement glorifier un après 60 étatiste qui est loin d'être chromée ${ }^{29}$.

24 Ibid., p. 52.

25 Ibid., p. 72.

${ }^{26}$ Voir, à ce propos, Gilles Bourque, "Parti pris : l'indépendance, la laïcité et le socialisme ", dans Robert Comeau, Charles-Philippe Courtois et Denis Monière (dir.), Histoire intellectuelle de l'indépendantisme québécois, t. 1 : 1834-1968, Montréal, VLB éditeur, 2010, p. 198-207.

${ }_{27}$ Courtois, "Cité libre, Duplessis ", p. 65.

28 Gilles Paquet, "Révolution tranquille et gouvernance : trois chantiers - éducation, santé et culture ", dans Guy Berthiaume et Claude Corbo (dir.), La Révolution tranquille en héritage, Montréal, Éditions du Boréal, 2011, p. 79.

29 Ibid., p. 77. 
Faut-il souligner que nous sommes ici et de plain-pied sur le terrain politique. Que font de l'idée de Grande Noirceur les auteurs auxquels nous nous sommes référés : les uns s'adonnent à la réitération comme si la notion avait produit un savoir non questionnable; les autres, adeptes de la rectification, s'empressent de la contester et cherchent à en montrer la fausseté. Or, dans les deux cas, ils paraissent prendre la notion au pied de la lettre ou, pourrait-on dire, au premier degré. D'un côté, le camp du Vrai, de l'autre, celui du Faux. Ils semblent incapables de construire l'objet en dehors de son prétendu rapport à la vérité.

En conséquence, les auteurs demeurent enfermés dans l'espace discursif créé et délimité par la notion de Grande Noirceur. D'abord, en se limitant à la seule question de la vérité et de la fausseté, on se condamne soit à l'éternelle réitération, soit à une entreprise de rectification qui finit par sombrer dans les affres d'une quelconque théorie du complot, conception élémentaire, faut-il le dire, des rapports de force idéologiques. Plus encore, les auteurs se piègent eux-mêmes dans les mailles du discours. Je soutiendrai plus bas que l'idée de Grande Noirceur participe à une formation discursive beaucoup plus large qui tient lieu de discours politique dominant durant les années 1960 et 1970 . En ce sens, quand on se contente de répéter ou d'invalider l'idée de Grande Noirceur, on fait au sens strict de la politique ou, dit autrement, on produit soi-même du discours politique : il s'agirait d'être pour ou contre Duplessis.

\section{L'objectivation}

D'autres auteurs tentent d'appliquer les règles de la méthode scientifique dans les domaines des sciences sociales, la première de toutes étant certainement celle de l'objectivation. Celle-ci met en œuvre une démarche de construction de l'objet qui implique un travail théorique permettant la définition de concepts et le développement de méthodes de recherche claires et reproductibles. Dans cette courte section, je m'en tiendrai aux concepts produits par les auteurs qui, implicitement ou explicitement, obéissent aux règles de 
l'objectivation. Comme il fallait s'y attendre, la conceptualisation differe selon les auteurs. J'ai ainsi repéré les notions de tropes, de métadiscours, de récit, d'imaginaire, de mythe, de discours et de mythistoire. Le concept de mythe demeure certainement le plus employé, mais dans une perspective différente. Les adeptes de la rectification, nous l'avons vu, emploient de façon univoque l'idée de mythe dans le sens commun d'invention et de contrevérité. Sous cet angle, il ne reste plus qu'à rétablir les « faits » et à montrer du doigt les coupables.

Tout autre devient la notion de mythe lorsqu'elle résulte d'un travail de conceptualisation qui permet d'en faire un concept clairement défini, lequel s'inscrit dans une théorie plus large de la représentation du monde, ce qui est clairement le cas dans les travaux de Gérard Bouchard. Commençons par sa définition du concept de mythe. Pour Bouchard, ce concept réfère à " une instauration durable de valeurs et de sens (entretenant ou non un rapport avec le sacré) qui commande d'autres valeurs et d'autres sens, qui fixe des règles à l'identité et une direction à l'action collective, qui s'incarne, enfin, aussi bien dans des objets et des personnages que dans des évènements et des récits ${ }^{30}$ ». Il importe de le souligner, pour Bouchard, le mythe ne se suffit pas à lui-même. Il se rapporte à une théorisation plus large qui englobe les concepts d'imaginaire collectif et de discours. Nous ne nous en tiendrons ici qu'à la question du rapport du mythe à la vérité. Bouchard écrit à ce propos : «[L]e mythe doit être évalué sous le rapport de l'efficacité symbolique autant que de la vérité intrinsèque. Sous cet éclairage on a donc tort de le tenir pour une simple chimère ou une falsification; il est plutôt l'affirmation plus ou moins opérante d'un jeu de représentation ${ }^{31}$ ". Pour l'auteur, le rapport à la vérité ne représente ainsi que l'un des aspects de l'analyse

${ }^{30}$ Gérard Bouchard, "L'imaginaire de la grande noirceur et de la révolution tranquille : fictions identitaires et jeux de mémoire au Québec ", Recherches sociographiques, vol. 46, $\mathrm{n}^{\circ} 3$ (2005), p. 413.

31 Ibid. 
d'un mythe, contrairement aux tenants de la rectification présentée plus haut.

Rappelons que cette question du rapport à la vérité représentait une préoccupation centrale dans les travaux sur le discours et les idéologies durant les années 1960 et 1970. Il s’agissait de rompre avec cette idée surannée que l'idéologie n'est qu'un reflet inversé des rapports réels, sinon une simple invention ou une pure falsification produites par les classes dominantes ${ }^{32}$. Cette même question est reprise par Jocelyn Létourneau et Alexandre Turgeon autour des concepts de mythe et de mythistoire. Selon Létourneau, le mythistoire peut être défini comme " tout à la fois une fiction réaliste, un système d'explication et un message mobilisateur qui rencontre une demande de sens, si ce n'est un désir de croyance chez ses destinataires ${ }^{33} »$. Pour sa part, Turgeon, à partir du jeu entre le vrai, le fictif et le faux, tente de distinguer entre " le mythe où prime le fictif et le mythistoire où prime le faux ${ }^{34} »$. Dans une telle perspective, il considère la Grande Noirceur duplessiste comme un mythistoire. Il tient cependant à insister sur le fait qu' une telle approche ne conduit pas à une entreprise de rectification : " Il ne s’agit pas de démasquer le faux, les faussetés ou les faussaires pour corriger "une erreur" qui aurait été commise, mais plutôt de comprendre ce qu'il en a été et ce qu'il est ${ }^{35}$ ».

Malgré leur intérêt, je ne pourrai discuter sérieusement des propositions conceptuelles de Bouchard, de Létourneau et de Turgeon. Il ne s'agissait ici que de montrer comment l'acte de compréhension ou d'interprétation résulte d'un travail théorique et, dans toute son

32 Voir, à ce propos, Gilles Bourque et Jules Duchastel, "Montée et déclin de l'idéologie au Québec ", Les Cahiers Fernand Dumont pour la mémoire, n 3 (hiver 2014).

33 Jocelyn Létourneau, « Mythistoires de Losers : introduction au roman historial des Québécois d'héritage canadien-français ", Histoire sociale = Social History, vol. 39, nº 77 (mai 2006), p. 162.

${ }^{34}$ Alexandre Turgeon, " "Toé, tais-toé!" et la Grande Noirceur duplessiste : genèse d'un mythistoire ", Histoire sociale = Social History, vol. 56, n 92 (novembre 2013), p. 369.

35 Ibid., p. 368. 
extension, de l'application de règles de recherche explicites et reproductibles.

\section{La Grande Noirceur et la Révolution tranquille}

Dans cette deuxième partie, je proposerai de déplacer le regard vers les années 1960 et 1970 . Il ne s'agit plus de commencer par nous demander si la notion dit vrai à propos des années 1950, mais de nous interroger sur la participation de l'idée de Grande Noirceur à la production de la représentation du monde qui dominera à partir de la Révolution tranquille jusqu'au début des années 1980. Cela implique, à l'évidence, une façon différente de construire l'objet puisqu'il nous faudra dévoiler la place et le jeu des relations qu'entretenait la notion de Grande Noirceur dans l'espace discursif de la période. En m’inspirant du cadre théorique que j'ai développé avec Jules Duchastel dans nos recherches sur le duplessisme avec la collaboration de Jacques Beauchemin, je ne proposerai que des pistes de recherche et une esquisse de problématique ${ }^{36}$.

\section{Quelques concepts}

Je définirai d'abord le plus succinctement possible les concepts nécessaires à la compréhension de l'exercice que je propose, soit ceux de discours politique, d'imaginaire, d'État moderne et de forme de l'État. J'entends par le concept d'État moderne ce complexe d'institutions construit à la suite du renversement de l'État absolutiste en Europe et de l'État colonial en Amérique, qui trouvera sa forme achevée sous les traits de la démocratie libérale. L'État moderne détient et exerce la pleine et entière souveraineté et, à ce titre, le monopole de la violence légitime encadrée par le droit. Une de ses caractéristiques principales est de procéder à la séparation de la société en deux espaces

36 Bourque, Duchastel et Beauchemin, La société libérale duplessiste; Bourque et Duchastel, Restons traditionnels et progressifs; Jacques Beauchemin, Gilles Bourque et Jules Duchastel, «Du providentialisme au néolibéralisme, de Marsh à Axworthy: un nouveau discours de légitimation ", Cahiers de recherche sociologique, $n^{\circ} 24,1995$. 
institutionnels : la sphère publique et la sphère privée. Dernière remarque essentielle à notre propos : le pouvoir politique moderne ne peut se réclamer d'aucune force extérieure susceptible d'assurer sa légitimité comme dans les monarchies de droit divin (ce qui ne veut pas dire que la religion et les institutions religieuses n'interviennent pas dans l'institutionnalisation de la société). C'est plutôt au nom de la souveraineté nationale populaire qu'on exercera le pouvoir. Cette dernière caractéristique implique l'existence d'une formation discursive assurant la légitimation du pouvoir dans l'État démocratique : le discours politique.

L'État moderne, bien sûr, se transforme dans le temps et à la faveur des luttes sociales. C'est pour saisir le sens de ces transformations qu'avec d'autres nous avons proposé le concept de forme de l'État. Ce concept permet de dégager la logique générale de la régulation politique durant une période de l'histoire de l'État moderne (par exemple, l'État libéral, l'État-providence, l'État néolibéral). La forme de l'État-providence qui nous intéresse davantage ici se caractérise par une régulation politique dominée par la sphère publique et vouée au développement du marché dans le cadre national grâce au soutien et à la stimulation de la demande.

Par ailleurs, le discours politique représente l'une des formations discursives les plus caractéristiques de la modernité en ce qu'il assure, sur le plan symbolique, l'exercice du pouvoir dans les démocraties libérales. Au sens restreint, le concept vise les discours émanant des institutions publiques, des partis et de la classe politiques. Au sens large, il inclut tout discours qui intervient sur les conditions de production de la société et, à ce titre, contribue à la représentation des grands repères anthropologiques que sont le temps, l'espace, la communauté, les rapports sociaux ainsi que la place et le rôle de l'individu-citoyen dans cette même société (par exemple, les dimensions éthiques ${ }^{37}$. Le discours peut émaner aussi bien de la sphère privée que de la sphère publique, voire être avant tout d'ordre

37 Jacques Beauchemin, "Les formes de l'État et la production de l'éthique sociale dans la perspective de la sociologie politique », Politique et Sociétés, vol. 16, 1997. 
économique (un économiste et une organisation patronale qui prônent le primat du marché), ou d'ordre culturel (des écrivains qui militent pour l'avènement d'une littérature nationale ${ }^{38}$. J'entendrai enfin par imaginaire un ensemble de présupposés cognitifs qui découlent de la formulation, de la répétition et de la domination d'un discours politique durant une période de l'histoire d'une société. Cet imaginaire prédispose " tout naturellement " l'individu soumis à ce discours à privilégier un certain type de rapports à soi et à l'autre et l'invite à répondre d'une certaine manière aux questions qui se posent dans la société. Je ne prendrai pour exemple que cette réaction quasi viscérale qui s'empare de bon nombre de Québécois francophones qui ont vécu les années 1950 et la Révolution tranquille avant même que puisse s'engager une discussion rationnelle sur la question du voile islamique.

\section{Le contexte}

Avant de commencer l'analyse de ce que j'appellerai le discours providentialiste québécois, il ne sera pas inutile de faire un très bref rappel du contexte qui favorisa l'avènement de la Révolution tranquille. Je ne retiendrai que trois dimensions de ce contexte. La Révolution tranquille marque d'abord l'affirmation de l'Étatprovidence au Québec, processus de transformation de l'État qui avait commencé dans l'après-guerre au niveau fédéral et auquel le régime Duplessis avait résisté jusqu'à la fin. Le providentialisme, nous l'avons vu, privilégie la mise en œuvre d'une régulation politique centrée sur la sphère publique, ce qui s'est traduit par la création d'une pléthore d'institutions : sociétés d'État, fonction publique d'inspiration wébérienne, ministère de l'Éducation, réseau et système d'aide sociale. Comme deuxième dimension, il faut rappeler les transformations du capitalisme dans l'après-guerre, caractérisées par le développement du fordisme (généralisation du taylorisme liée à

38 Voir Jacques Godbout, "Du poétique au politique », dans Berthiaume et Corbo (dir.), La Révolution tranquille en héritage, p. 271-296. 
celle du régime de la convention collective) et de la société de consommation. Retenons enfin, à partir de la fin de la Deuxième Guerre mondiale, la formation progressive d'une nouvelle classe moyenne, fascinée par la société de consommation, la culture hédoniste et la révolution des mœurs ${ }^{39}$ qu'elle impliquait. L'Étatprovidence profitera à cette nouvelle classe : durant la Révolution tranquille s'ouvriront un très grand nombre de postes dans les institutions publiques en même temps que le régime de la convention collective exercera une pression à la hausse sur les salaires.

\section{Le discours providentialiste québécois}

Puisqu'aucun parti n'a dominé durant toute la période, comme l'Union nationale de 1944 à 1960, il nous faudra un concept moins ciblé que celui de discours duplessiste pour nommer le discours politique dominant durant les années 1960 et 1970. J'ai choisi de retenir la particularité de la régulation politique qui permet de caractériser cette vingtaine d'années, soit le providentialisme. Le qualificatif québécois tient ici lieu de marqueur de spécificité, puisque, bien sûr, ni le discours providentialiste ni l'État-providence ne se sont développés de la même manière dans tous les pays. On s'étonnera peut-être que je retienne le début des années 1980 comme limite temporelle de la période. C'est qu'on assiste dès lors au démantèlement progressif de l'État-providence jusqu’à la formation de l'État néolibéral durant la décennie 1990, en même temps que le discours se transforme à l'avenant ${ }^{40}$.

39 Jean-Marc Piotte, La révolution des mours: comment les baby-boomers ont changé le Québec, Montréal, Québec Amérique, 2016.

${ }^{40}$ Gilles Bourque, "Prolégomènes à l'analyse des transformations de la régulation politique au Québec », dans Raphaël Canet et Jules Duchastel (dir.), La régulation néolibérale : crise ou ajustement?, Montréal, Athéna éditions, 2004. 


\section{Un discours binaire}

Le discours providentialiste québécois s'organise autour de deux notions centrales : la Grande Noirceur et la Révolution tranquille. Il est sans doute pertinent d'insister sur le fait que l'idée de Révolution tranquille constitue une notion (ou un mythe) au même titre que celle de Grande Noirceur. Inventée, paraît-il, par un journaliste anglophone, la notion de Révolution tranquille s'est répandue comme une traînée de poudre dans tous les recoins imaginables de la production discursive québécoise. On peut cependant avancer qu'elle n'aurait pu s'imposer de telle manière sans sa contrepartie, le repoussoir que représentera l'invention de la Grande Noirceur. En somme, les notions de Grande Noirceur et de Révolution tranquille se nourrissent mutuellement et chacune génère une chaîne de significations étrangère l'une à l'autre.

Cette double série obéit à une seule et même dynamique dont la contradiction constitue le principe de déploiement. Le discours providentialiste québécois produit une structure notionnelle non seulement binaire, mais aussi parfaitement et " éternellement " contradictoire. La double chaîne de significations ne saurait générer autre chose que des oppositions irréconciliables. Bref, il n'y a pas et il n'y aura jamais de synthèse possible entre la Grande Noirceur et la Révolution tranquille dans le discours providentialiste. La thèse de l'accès à la modernité au Québec en 1960, par exemple, cette sorte de retraduction dans les termes de sciences sociales de la notion de Révolution tranquille, ferme en quelque sorte les effluves du temps passé qui s'en trouvent réifiées alors même qu'elle instaure un temps présent aussi nouveau qu'ouvert sur l'avenir. Cette sorte d'hydre à deux têtes produit une chaîne de significations dont le principe de construction ne saurait être que la dichotomie. Je tenterai d'illustrer cette thèse à partir des dimensions du discours politique que j'ai évoquées plus haut, soit la représentation du temps, de l'espace, de la communauté, des rapports sociaux ainsi que des règles et des normes d'insertion de l'individu dans la société. Cette approche nous permettra de repérer et de décrire de façon schématique les anneaux 
de la chaîne des oppositions dichotomiques, des contradictions indépassables qui hantent le discours providentialiste québécois.

\section{La temporalité : le présent contre le passé}

Le discours providentialiste québécois entretient un rapport entièrement négatif avec le passé. L'idée d'une révolution (tranquille) indique d'ailleurs tout le mépris pour ce monde d'où l'on sort enfin. La volonté de rupture ne saurait être plus nette qu'à propos du Canada français.

Au-delà du fait que la période de l'après-guerre peut être représentée sous les traits de la Grande Noirceur, c'est finalement toute l'histoire du Québec qui sera décrite comme un échec. Le Québec d'alors aurait été une société attardée, restée enserrée dans les méandres caractéristiques d'un autre âge de l'histoire des sociétés occidentales : la prémodernité, le Moyen Âge, la société traditionnelle. Le chanoine Lionel Groulx devient la tête de Turc dans ce discours qui vient rompre avec sa conception surannée de l'histoire du Québec. Le passé ne saurait jamais plus être considéré comme "notre maître ", et la tradition se transforme en une vieillerie sur laquelle on peut à la rigueur s'attendrir en écoutant du folklore ou en achetant des antiquités qui, d'ailleurs, deviennent québécoises plutôt que canadiennes-françaises. Cette conception du passé relève de ce que Gérard Bouchard appelle une mémoire " honteuse ${ }^{41}$ ». Cette histoire et ce passé incarnent une sorte de manque constitutif, résultat d'une absence de mythe fondateur dont on pourrait s'enorgueillir et, surtout, à partir duquel on pourrait bâtir plutôt que de faire table rase.

La représentation du temps, dans la foulée de la notion de Révolution tranquille, rompt ainsi radicalement avec le passé. Elle se décline en deux moments. Celui du présent d'abord, c'est-à-dire celui de la rupture. On pense, à coup sûr, ici au fameux "désormais " (réel ou retraduit) de Paul Sauvé. Ce temps présent de la rupture ne

${ }^{41}$ Bouchard, "L'imaginaire de la grande noirceur et de la révolution tranquille ", p. 424. 
vaut que pour autant qu'il ouvre sur l'avenir. Nous sommes ainsi face à une conception essentiellement transformationniste de la temporalité comme, par exemple, dans la Ligne du risque de Pierre Vadeboncoeur ${ }^{42}$. Notons à ce propos le projet de devenir "Maîtres chez nous " aux élections de 1962, la volonté de réaliser la souveraineté-association, ou encore l'horizon du socialisme. Alors qu'il était ministre libéral durant la Révolution tranquille, René Lévesque aurait répondu à un journaliste qui lui demandait dans quelle direction s'orientait le gouvernement : "On ne le sait pas mais on y va vite ». Ce qui à vrai dire était rigoureusement exact. En quelques années, de 1960 à 1966, on créa l'essentiel du complexe institutionnel d'un État-providence que l'on peaufina ensuite sous les gouvernements de Daniel Johnson et de Robert Bourassa, alors qu'enfin, durant le premier mandat du gouvernement Lévesque de 1976 à 1980, la frénésie des réformes reprit de plus belle. La temporalité providentialiste n'avait cure du passé et ne se suffisait guère du présent.

\section{L'espace : les frontières, le territoire}

On peut analyser de deux manières la contribution du discours politique à la représentation de l'espace. La première cible est ce que l'on pourrait appeler la fixation des frontières, c'est-à-dire l'espace géographique à l'intérieur duquel s'exerce le pouvoir politique. La seconde interroge le rapport de l'État à la société. On pense ici à l'aménagement du territoire et, plus globalement, à la relation entre le pouvoir politique et le citoyen.

Commençons par l'espace territorial. Deux questions récurrentes hantent le discours politique au Québec depuis l'obtention d'une chambre d'assemblée au Bas-Canada en 1791 : au nom de qui et dans quel espace territorial s'exerce le pouvoir? Ne retenons provisoirement que la seconde. Différentes réponses ont été successivement

${ }^{42}$ Voir, à ce propos, Yvan Lamonde, La modernité au Québec : la victoire différée du présent sur le passé, t. 2 : 1939-1965, Montréal, Éditions Fides, 2016, p. 360-380, en particulier. 
données : le Bas-Canada (dans ou hors de l'Empire britannique), le Canada-Uni, le Canada et la province de Québec, le Québec indépendant. Si on peut repérer une faille dans le mur qui sépare les notions de Grande Noirceur et de Révolution tranquille, on la trouvera certainement dans la représentation de l'espace territorial. Il existe à ce propos un non-dit, une sorte de tabou qui colmate la brèche. Cette faille, c'est bien celle du thème de l'autonomie provinciale chère au chef de l'Union nationale. Si d'aventure il fallait l'évoquer, c'était bien davantage pour dénoncer son caractère défensif en ce qui a trait au pouvoir du Québec et passéiste dans son refus de l'État-providence. De la même manière, on reprend le slogan « Maîtres chez nous » sans faire référence aux années 1950. Dans les faits, le discours politique duplessiste accentue la tendance à la centration de la représentation dans l'espace du Québec ${ }^{43}$. Sur le plan de la représentation de l'espace territorial, la véritable césure s’opère ailleurs, dans le délestage de la référence au Canada français à titre d'espace politique significatif pour le Québec.

Une rupture très nette apparaît, au contraire, si l'on envisage ce deuxième aspect de la représentation de l'espace que j'ai appelé le rapport de l'État à la société. La notion de Grande Noirceur évoque les traits d'une régulation politique libérale autoritaire et patriarcale qui repose sur une alliance entre l'État provincial et l'Église catholique : elle organise et quadrille l'espace social à partir du comté et de la paroisse. L'idée de Révolution tranquille renvoie, pour sa part, à une régulation d'inspiration keynésienne qui exclut l'Église au profit de la formation d'une bureaucratie, laquelle procédera à une redéfinition du marquage du territoire en régions administratives, réforme d'ailleurs plusieurs fois revisitée à partir de règles techno-bureaucratiques qui transforment le citoyen en client des appareils étatiques.

${ }^{43}$ Voir Bourque, Duchastel et Beauchemin, La société libérale duplessiste, p. 226-250. 


\section{La communauté : de la nation canadienne-française à la nation québécoise}

Nous avons souligné plus haut que deux questions hantent l'histoire politique du Québec depuis 1791. Au nom de qui s'exerce le pouvoir et dans quel espace politique particulier? Il nous reste à aborder la première question, interrogation sans laquelle il n'y aurait aucun exercice légitime d'un pouvoir politique se réclamant de la démocratie libérale. Dans l'État moderne, la nation représente la communauté politique au nom de laquelle s'exerce le pouvoir. $\mathrm{Au}$ Québec, la désignation de la communauté nationale se transforme en même temps que la référence à l'espace politique. À la nation canadienne des Patriotes a succédé « la race » ou la nation canadiennefrançaise et, finalement, la nation québécoise. La notion de Grande Noirceur renvoie à ce temps honni du Canada français et de la nation canadienne-française. Elle cible la classe politique régnante et l'Église catholique qui entretenaient le mythe que la nation canadiennefrançaise allait s'étendre progressivement jusqu'à convertir l'Amérique. Bien que le discours politique dominant sous le régime Duplessis ait manifestement abandonné cette utopie, il ne s'inspire pas moins du nationalisme canadien-français et de cette idée que la Constitution canadienne résulte d'un pacte sacré entre deux « races ». Au chapitre de la représentation de la communauté, le discours providentialiste québécois (et, avec lui, la notion de Révolution tranquille) rompt radicalement avec le Canada français lors des États généraux du Canada français en $1969^{44}$. En même temps, durant les années 1960, sans trop que l'on sache comment, s'affirme l'idée de nation québécoise, communauté nationale au nom de laquelle, dorénavant, s'exercera le pouvoir politique dans l'État du Québec et non dans la province du même nom. Ce que l'on a appelé dès lors le néonationalisme imprégnera le discours de tous les partis, à tout le moins sur la scène provinciale, du " Maittres chez nous » du Parti

${ }^{44}$ Les États généraux du Canada français furent tenus à Montréal de 1966 à 1969. Ils avaient pour objectif de consulter ce que l'on appelait encore le peuple canadien-français. 
libéral, au projet "Égalité ou indépendance » de l'Union nationale à la "souveraineté-association " du Parti québécois, jusqu’au référendum perdu en 1980 .

\section{Les rapports sociaux : les Anciens et les Modernes}

S'il fallait trouver un domaine du discours providentialiste québécois où la césure entre la notion de Grande Noirceur et celle de Révolution tranquille est la plus radicale, il faudrait sans doute choisir celui de la représentation des rapports sociaux. La Révolution tranquille célèbre ici l'accès à la modernité, c'est-à-dire rien de moins que l'avènement de la société moderne au Québec.

La notion de Grande Noirceur dessine, au contraire, l'image d'une société traditionnelle ou d'une société paysanne. Pourtant, durant cette période et depuis longtemps, l'économie du Québec est dominée par le marché et le capitalisme, la société de consommation s'y développe comme partout en Amérique du Nord et plus rapidement qu'en Europe. Finalement, malgré toutes les nuances que l'on pourra apporter, dans le cadre du fédéralisme canadien, l'État provincial demeure un État de droit. Ce constat visait à cibler de façon plus précise ce que la notion de Grande Noirceur permet de honnir dans le Québec de l'après-guerre. Énumérons à travers elle certains traits qui suscitent la répulsion : la conception hiérarchique des rapports sociaux, l'autoritarisme institué, la centralité de la famille élargie de nature patriarcale et, surtout, la dominance de l'Église catholique dans la régulation sociale : la santé, l'éducation, l'aide sociale. Or, c'est précisément ce que Jürgen Habermas a appelé les " réserves de traditions ». Selon l'auteur, les sociétés libérales occidentales ont pu se reproduire pendant plus d'un siècle grâce à la place qu'ont occupée des institutions traditionnelles comme l'Église et la famille patriarcale dans la sphère privée ${ }^{45}$. C'est ce lien jugé délétère que cible la notion de Grande Noirceur.

45 Jürgen Habermas, L'espace public : archéologie de la publicité comme dimension constitutive de la société bourgeoise, Paris, Éditions Payot, 1978. 
Avec l'idée de Révolution tranquille et ses allures de Grand Soir, le discours providentialiste québécois vise d'abord et avant tout l'avènement de la centralité de la sphère publique dans la régulation politique. Si l'on parle désormais d'État du Québec plutôt que de province de Québec, il faut l'entendre de deux manières : le Québec d'abord devient un lieu déterminant de pouvoir face au fédéral, il s'agit d'un État au même titre que l'État canadien; la locution, ensuite, renvoie à la place centrale de la sphère publique dans la régulation politique, rendant ainsi caduc le rôle de l'Église. La notion de Révolution (tranquille) révèle aussi ce second aspect du discours providentialiste québécois, que j'appellerai la propension à la politisation de tous les pouvoirs. Le discours providentialiste et, bien sûr, l'idée de révolution invite à la compréhension des relations entre les groupes sociaux et avec l'État comme des rapports de force, de pouvoir et de domination. D'une certaine manière, le discours providentialiste peut-être retourné contre lui-même. La question n'est pas de savoir ici si la cause est juste ou injuste, mais de noter que ce discours ouvertement politique et centré sur l'État ouvre la possibilité de la politisation du rapport salarial, des rapports de classe, des rapports de genre et, par l'intermédiaire des mouvements sociaux, celle de toutes les formes de micropouvoir.

\section{L'individu et la société}

Je terminerai ce survol de la contribution du discours providentialiste à la représentation des grands repères anthropologiques en traitant de la figure de l'individu proposée par les notions de Grande Noirceur et de Révolution tranquille. Nous sommes en terrain connu, aussi me permettrai-je d'être encore plus bref à ce chapitre.

La notion de Grande Noirceur renvoie à l'image d'un individu enserré dans les mailles d'un tissu social " tricoté serré », comme on le disait à l'époque. Dès la naissance, il paraît suivre une trajectoire programmée, familiale, religieuse et politique, de laquelle il est extrêmement problématique de s'échapper. La Grande Noirceur 
rappelle évidemment le Québec duplessiste, son discours autoritaire axé sur l'ordre et les devoirs (avant les droits) et la disciplinarisation généralisée imposée par le discours religieux.

La Révolution tranquille invite, au contraire, l'individu à se délester de ses amarres familiales et religieuses. Il est inutile de revenir ici sur la littérature qui porte sur l'individualisme, la révolution des mœurs ${ }^{46}$ et l'hédonisme de la génération lyrique ${ }^{47}$. La Révolution tranquille propose l'affirmation des droits à l'encontre des pouvoirs aliénants. On oublie trop souvent cependant que le discours providentialiste québécois, entre 1960 et 1980, invitait en même temps à œuvrer pour l'égalité et la justice sociale, valeurs qui furent portées par un grand nombre.

À la fin de cet inventaire fort sommaire, il faut le souligner, on peut donc dégager deux chaînes de significations parfaitement contradictoires, comme si un seul et même discours référait à deux mondes étrangers l'un à l'autre et, surtout, parfaitement antithétiques. D'un côté, la Grande Noirceur : le passé, la tradition, la province, le Canada français, la nation canadienne-française, le Moyen Âge, la famille, l'Église, l'autorité, la hiérarchie; de l'autre, la Révolution tranquille, le présent, l'avenir, l'État, le Québec, la nation québécoise, la société moderne, les rapports de force, l'individu, l'égalité, la justice sociale.

\section{Un imaginaire d'émancipation}

Reprenons d'abord, en la précisant, la définition du concept d'imaginaire que j'ai proposée plus haut. J'entends par imaginaire un ensemble de présupposés qui prédisposent un individu, un groupe ou une collectivité à adopter un point de vue particulier sur les rapports sociaux; une telle présupposition l'amènera soit à poser un jugement spontané, soit, au contraire, à développer un argumentaire rationnel à propos d'une situation donnée. Cet imaginaire résulte de

46 Piotte, La révolution des mours.

47 François Ricard, La génération lyrique : essai sur la vie et l'œuvre des premiers-nés du baby-boom, Montréal, Éditions du Boréal, 1984. 
l'interaction entre une pluralité de discours. Je tenterai, en terminant, de faire ressortir quelques-uns des traits principaux de l'imaginaire induit par le discours providentialiste québécois.

L'imaginaire providentialiste se caractérise d'abord et avant tout par une prédisposition "naturelle " à l'émancipation. C'est à partir de la contradiction centrale (et non synthétisable, nous l'avons vu) du discours providentialiste québécois entre les notions de Grande Noirceur et de Révolution tranquille que l'on doit comprendre l'émergence et l'affirmation de cet imaginaire. La Grande Noirceur désigne ce monde du passé duquel il faut absolument sortir, dont il faut s'émanciper. Du seul fait qu'il n'y ait pas de synthèse possible entre les notions centrales du discours, entre le passé et le présent, la Révolution tranquille, l'émancipation en acte en quelque sorte, se retourne contre elle-même, en incitant à des pratiques libératrices sans cesse renouvelées. Dit autrement, le présent de la Révolution se transforme en cet avenir habité par l'espoir d'une émancipation permanente. Et la Révolution tranquille sera la première à être accusée de ne pas avoir réalisé les espoirs qu'elle avait suscités ${ }^{48}$.

L'imaginaire providentialiste se donne comme un imaginaire de la rupture et de la désaffiliation. Ces caractéristiques ne sont, à vrai dire, que l'envers ou la condition des pratiques émancipatrices. Il faut d'abord rompre pour ouvrir la voie de l'émancipation : avec le Canada, afin de libérer la nation; avec le capitalisme, condition de la réalisation du socialisme... Il importe aussi de se désaffilier (de la famille, du passé, du patriarcat...) si l'on entend se réaliser de façon pleine et entière.

\footnotetext{
${ }^{48}$ Rappelons, à ce propos, la publication du manifeste Ne comptons que sur nos propres moyens, par la Confédération des syndicats nationaux en 1971 et, la même année, celle de L'État, rouage de notre exploitation, par la Fédération des travailleurs du Québec, de même que la parution, en 1974, du document de la Centrale de l'enseignement du Québec, École et lutte de classes au Québec.
} 


\section{Et le duplessisme}

La notion de Grande Noirceur, ai-je soutenu, devrait d'abord être considérée comme une des deux notions centrales du discours providentialiste québécois qui a dominé la représentation politique durant les années 1960 et 1970 . Cela ne veut certes pas dire qu'il ne soit pas pertinent de travailler à une histoire de l'idée de Grande Noirceur dès lors qu'elle devient un objet soumis aux règles de la recherche. Les très intéressants travaux de Turgeon sont précieux à cet égard ${ }^{49}$. Celui-ci montre comment le célèbre "Toé, tais-toé! " représente un mythistoire dont le développement relève manifestement du journalisme. L'histoire de la production de la notion de Grande Noirceur, que Turgeon considère aussi comme un mythistoire, reste à faire. On peut cependant penser que cette notion, résultat de l'amalgame d'une multiplicité de discours de niveaux différents, pourrait être aussi une métaphore médiatique comme sa sœur ennemie, la Révolution tranquille.

Les travaux de Turgeon s'inscrivent dans le cadre plus large d'une histoire des idées respectueuse du déroulement historique, qui ne confond pas le discours critique de l'opposition au régime Duplessis durant les années 1950 avec la caricature qu'on voudra en faire plus tard sous la notion de Grande Noirceur. Les travaux d'Yvan Lamonde se révèlent exemplaires à ce propos ${ }^{50}$. Seule l'accumulation de travaux empiriques permettra de faire progresser les connaissances et d'aider à comprendre les particularités du régime et de la société québécoise de l'après-guerre jusqu'en 1960. Dans La société libérale duplessiste, nous avons tenté de montrer comment cette société se produisait à l'ombre de l'État libéral, soumise à une régulation politique de nature autoritaire et disciplinaire favorisant le libre marché et les institutions privées (principalement l'Église catholique) dans les domaines social et culturel $^{51}$. Plus récemment, Jean-Philippe Warren a proposé de

\footnotetext{
49 Voir, en particulier, Turgeon, "“Toé, tais-toé!” et la Grande Noirceur duplessiste ».

50 Lamonde, La modernité au Québec.

51 Bourque, Duchastel et Beauchemin, La société libérale duplessiste.
} 
considérer le duplessisme comme un régime liant autoritarisme et pratiques démocratiques. Pour lui, " les régimes autoritaro-démocratiques sont, en effet, des types de gouvernance qui, tout en permettant en théorie sinon en fait l'alternance, reposent en partie sur la fraude, la violation des libertés, la censure des médias et l'abus des ressources démocratiques ${ }^{52}$ ".

Le concept d'autoritarisme démocratique proposé par Warren, malgré son intérêt, n’en est pas moins beaucoup trop large, car il finit par englober aussi bien le duplessisme que le régime Poutine en Russie. Ainsi se retrouvent amalgamés une gouverne qui s'inscrivait dans l'histoire de la démocratie libérale canadienne depuis le XIX ${ }^{\mathrm{e}}$ siècle et un pouvoir politique qui hérite de la culture totalitaire de l'exURSS, s'appuie sur l'armée et assure la domination des oligarques. S’il fallait retenir le concept, il faudrait en revoir la définition afin de cibler de façon plus spécifique des expériences historiques similaires. Ainsi, pourrait-on peut-être accoler l'appellation d'autoritarisme démocratique à plusieurs pouvoirs politiques régionaux qui, dans l'histoire de l'État libéral aux États-Unis comme au Canada et en Europe, ont lié de manière autoritaire, quoique de façon formellement démocratique, la promotion du libre marché et la défense des institutions traditionnelles (les Églises, la famille élargie) dans la régulation des rapports sociaux.

Quoi qu'il en soit de ce type de discussion, la notion de Grande Noirceur paraîtra toujours mauvaise conseillère dans l'analyse du duplessisme. Comme je l'ai rappelé dans l'introduction, lors de la publication de nos travaux sur les années 1940 et 1950, nous avons été soupçonnés de chercher à réhabiliter le duplessisme. En boutade, Jules Duchastel et moi répondions qu'il n'était rien que nous voulions revivre de cette période hormis notre jeunesse. À la fin de cet article, certains pourraient m'accuser de contribuer à noircir la Révolution

52 Jean-Philippe Warren, « Note de recherche. Religion et politique dans les années 1950 : une pièce de plus à notre compréhension de la supposée Grande Noirceur ", Revue d'histoire de l'Amérique française, vol. 67, nos 3-4 (2014), p. 408. 
tranquille. Pourtant, je revivrais chaque moment des années 1960 et 1970 et je demeure, sans nul doute, imprégné de l'imaginaire d'émancipation qui les a caractérisées ${ }^{53}$.

53 Gilles Bourque, "La Révolution tranquille : entre les velléités de l'oubli et les impératifs de la mémoire ", dans Yves Bélanger, Robert Comeau et Céline Métivier (dir.), La Révolution tranquille 40 ans plus tard: un bilan, Montréal, VLB éditeur, 2000, p. 107-119. 